\title{
Near-Infrared Monitoring of Volatiles in Frozen Lunar Simulants While Drilling
}

Ted L. Roush ${ }^{1}$ Anthony Colaprete ${ }^{2}$, Richard C. Elphic ${ }^{3}$, Joshua Forgione ${ }^{4}$, Bruce White ${ }^{5}$, and Robert McMurray ${ }^{6}$ NASA Ames Research Center, Moffett Field, CA, 94035-1000

Amanda M. Cook ${ }^{7}$, Richard Bielawski ${ }^{8}$, and Erin Fritzler ${ }^{9}$ Millenium Engineering and Integration Company, Moffett Field, CA, 94035-1000

Sarah J. Thompson ${ }^{10}$

Intrinsyx Technologies Corporation, Moffett Field, CA, 94035-1000

Julie E. Kleinhenz ${ }^{11}$

NASA Glenn Research Center, Cleveland, OH, 44135

Joshua Benton ${ }^{12}$

Wyle Engineering, Houston, TX, 77058

Gale Paulsen ${ }^{13}$ and Kris Zacny ${ }^{14}$

Honeybee Robotics Spacecraft Mechanism Corporation, Pasadena, CA, 91103

James Smith $^{15}$

NASA Kennedy Space Center, FL, 32899

${ }^{1}$ NIRVSS Instrument Scientist, Planetary Systems Branch, Mail Stop 245-3, Moffett Field, CA, 94035-1000.

${ }^{2}$ NIRVSS Principal Investigator, Planetary Systems Branch, Mail Stop 245-3, Moffett Field, CA, 94035-1000.

${ }^{3}$ NSS Principal Investigator, Planetary Systems Branch, Mail Stop 245-3, Moffett Field, CA, 94035-1000.

${ }^{4}$ NIRVSS Electrical Engineer, Engineering Systems Division, Mail Stop 213-2, Moffett Field, CA, 94035-1000.

${ }^{5}$ NIRVSS LCS Design Lead, Engineering Systems Division, Mail Stop 213-2, Moffett Field, CA, 94035-1000.

${ }^{6}$ NIRVSS Engineer, Engineering Systems Division, Mail Stop 244-10, Moffett Field, CA, 94035-1000.

${ }^{7}$ NIRVSS Integration and Test Lead, Space Science and Astrobiology Division, Mail Stop 245-6, Moffett Field, CA, 94035-1000.

${ }^{8}$ NIRVSS Systems Engineer, Engineering Systems Division, Mail Stop 211-2, Moffett Field, CA, 94035-1000.

${ }^{9}$ NIRVSS Instrument Manager, Engineering Systems Division, Mail Stop 211-5, Moffett Field, CA, 94035-1000.

${ }^{10}$ NIRVSS DOC Design Lead, Intrinsyx Technologies Corporation, 350 N. Akron Road, Suite 2028, Moffett Field, CA, 94035-1000

${ }^{11}$ Research Engineer, Propellants and Propulsion Branch, MS 301-3 21000 Brookpark Road, Cleveland, OH 44135, Senior Member.

${ }_{12}$ NIRVSS Mechanical Engineer, Science Technology and Engineering Group, MS 213-3, Moffett Field, CA, 94035-1000.

${ }^{13}$ Senior Systems Engineer, 398 W Washington Blvd, Suite 200, Honeybee Robotics, Pasadena, CA, 91103.

${ }^{14}$ Director, Exploration Technology Group, 398 W Washington Blvd, Suite 200, Honeybee Robotics, Pasadena, CA, 91103, Senior Member.

${ }^{15}$ RESOLVE Lead Systems Engineer, Structures \& Mechanisms Design Branch, NE-M2, NASA Kennedy Space Center, FL, 32899 
In Situ Resource Utilization (ISRU) focuses on using local resources for mission consumables. The approach can reduce mission cost and risk. Lunar polar volatiles, e.g. water ice, have been detected via remote sensing measurements and represent a potential resource for both humans and propellant. The exact nature of the horizontal and depth distribution of the ice remains to be documented in situ. NASA's Resource Prospector mission (RP) is intended to investigate the polar volatiles using a rover, drill, and the RESOLVE science package. RP component level hardware is undergoing testing in relevant lunar conditions (cryovacuum). In March 2015 a series of drilling tests were undertaken using the Honeybee Robotics RP Drill, Near-Infrared Volatile Spectrometer System (NIRVSS), and sample capture mechanisms (SCM) inside a 'dirty' thermal vacuum chamber at the NASA Glenn Research Center. The goal of these tests was to investigate the ability of NIRVSS to monitor volatiles during drilling activities and assess delivery of soil sample transfer to the SCMs in order to elucidate the concept of operations associated with this regolith sampling method.

Ten soil samples were captured over the course of two tests at pressures of $10^{-7}-10^{-5}$ Torr and soil temperatures were $203^{\circ}$ to $213^{\circ} \mathrm{K}$ and $\approx 93^{\circ} \mathrm{K}$ for tests 1 and 2, respectively. Each sample was obtained from a depth of 30 to $40 \mathrm{~cm}$ within a cryogenically frozen layered bed of NU-LHT-3M lunar regolith simulant. The simulant was doped with 0.1 and $0.2 \mathrm{wt} \%$ water in the upper $\sim 16 \mathrm{~cm}$, for test 1 and 2 respectively, and $\sim 5 \mathrm{wt} \%$ of water below, for both tests, with the two layers separated by aluminum foil. For each sample the drilling sequence consisted of insertion and extraction of the drill to 10, 20,30, and $40 \mathrm{~cm}$ depths, and after the final depth the sample was delivered to the SCM. NIRVSS acquired spectra and images during the drilling sequence and these are the focus of the discussion here. NIRVSS spectra exhibit spectral features associated with water ice after the drill penetrated the aluminum barrier, and especially on extraction from $20 \mathrm{~cm}$ depth. These spectral features can be temporally correlated with measurements of the evolved water from the surface during the drilling sequence and images document the growth and evolution of the cuttings pile deposited by the drill. The spectra and images also document some anomalous behavior associated with the refilling of an existing hole due to the constraints imposed by the confined dimensions of the chamber.

\section{Nomenclature}

$\begin{array}{ll}\text { avg } & =\text { average } \\ B A & =\text { Bracket Assembly } \\ \text { BD\# } & =\text { Band Depth at wavelength \# } \\ \text { CMOS } & =\text { Complementary Metal-Oxide Semiconductor } \\ \text { COTS } & =\text { Commercial Off The Shelf } \\ D H & =\text { Drill Hole } \\ D N & =\text { Data Number } \\ D O C & =\text { Drill Observation Camera } \\ \text { EDU } & =\text { Engineering Development Unit } \\ \text { ETU } & =\text { Engineering Test Unit } \\ \text { FOV } & =\text { Field of View } \\ \text { GRC } & =\text { Glenn Research Center } \\ \text { IR } & =\text { Infrared } \\ \text { LAVA } & =\text { Lunar Advanced Volatile Analysis } \\ \text { LCROSS } & =\text { Lunar Crater Observation and Sensing Satellite } \\ \text { LCS } & =\text { Long-wavelength Calibration Sensor } \\ \text { LED } & =\text { Light Emitting Diode } \\ \text { LW } & =\text { Long Wavelength } \\ \text { NIRVSS } & =\text { Near-Infrared Volatile Spectrometer System } \\ \text { NSS } & =\text { Neutron Spectrometer System }\end{array}$




$\begin{array}{ll}\text { OVEN } & =\text { Oxygen and Volatile Extraction Node } \\ P & =\text { Peck } \\ r \# & =\text { radiance at wavelength \# } \\ R G A & =\text { Residual Gas Analyzer } \\ R P & =\text { Resource Prospector } \\ R E S O L V E & =\text { Regolith and Environment Science and Oxygen \& Lunar Volatile Extraction } \\ S C M & =\text { Sample Capture Mechanism } \\ S T & =\text { Soil Tube } \\ S W & =\text { Short Wavelength } \\ V F 13 & =\text { Vacuum Facility \#13 }\end{array}$

\section{Introduction}

WASA's Resource Prospector (RP) is intended to characterize the volatile distribution near, and in, a permanently shadowed region on the moon ${ }^{1}$. The major activities of RP will include prospecting for volatiles while traversing the lunar surface and characterizing sub-surface properties and volatiles via drilling. For prospecting, RP includes a Neutron Spectrometer System ${ }^{2}$ (NSS), and the Near-Infrared Volatile Spectrometer System $^{3}$ (NIRVSS). For sub-surface characterization of volatiles RP includes a drill ${ }^{4}$ to collect and deliver subsurface samples to the Oxygen Volatile Extraction $\operatorname{Node}^{5}$ (OVEN) where the samples are heated and any gases evolved are passed into the Lunar Advanced Volatile Analysis ${ }^{6}$ (LAVA) subsystem. NIRVSS RP goals are to observe the surface during rover traverses and at excavation sites monitoring for water, and other volatiles, to constrain mineralogical and geological context of the site, and to measure the surface and sub-surface temperatures. NIRVSS observations are intended to enable near-real time assessment of volatile content of samples exposed during the drilling activities and can be used to decide if a sample should be delivered to OVEN, and subsequently LAVA for more detailed analyses.

During March 2015 the NIRVSS engineering test unit (ETU), a Honeybee Robotics drill, and five sample capture mechanisms (SCMs) were placed in a NASA Glenn Research Center (GRC) cryo-vacuum facility (VF13) along with lunar simulant soil tubes prepared with varying amounts of water. The effort had the objective of simulating observations during drilling activities on the cold lunar surface and assessing effective delivery of soil samples for capture and sealing in the SCMs. Here we report the results of the NIRVSS measurements.

\section{Instrumental and Experimental}

\section{A. Instrumental}

\section{NIRVSS}

The NIRVSS ETU is an evolution from the NIRVSS EDU used in Hawaii ${ }^{3}$, which was a flight spare of the Lunar Crater Observation and Sensing Satellite (LCROSS) Hadamard transform near-infrared spectrometer ${ }^{7}$, but covering a different wavelength range. The NIRVSS ETU consists of two separate components. One component, the spectrometer assembly, is intended to be mounted on the RP science payload pallet located on the rover upper deck. It contains two near-infrared spectrometers, also referred to as optical engines, each fed by a separate fiber optic cable (Fig. 1), and associated electrical and data interfaces. The other component, the bracket assembly (BA), will be mounted on the bottom of the RP rover pointed downward. It contains two connections for the fiber optic cables, an associated infrared illumination source, a drill observation camera (DOC) with several LEDs as illumination sources, a Long-wavelength Calibration Sensor (LCS) to document surface and subsurface temperature, and electrical and data interfaces (Fig. 1).

In order to be sensitive to vibrational modes of water (near 2000 and $3000 \mathrm{~nm}$ ) and other volatile ices, electronic transitions associated with $\mathrm{Fe}^{2+}$ located in some igneous silicates, and vibrational modes of hydroxylated silicates (approximately 2200-2400 $\mathrm{nm}$ and near 2700-2800 nm), the two NIRVSS optical engines cover a different wavelength range than the LCROSS instrument. Both are COTS devices with modest changes for consistency with spaceflight hardware. The NIRVSS SW optical engine covers $\sim 1600-2400 \mathrm{~nm}$, with $\sim 9 \mathrm{~nm}$ sampling, and the NIRVSS LW optical engine covers $\sim 2300-3400 \mathrm{~nm}$ with $\sim 12 \mathrm{~nm}$ sampling. Each optical engine is attached to a fiberoptic cable with the other end of each cable connected to the BA that is designed to view the surface underneath the RP rover. Also located on the BA, and essential for spectrometer operation in shadowed regions on the moon, is the IR illumination source, located in the lamp assembly (Fig. 1) at the end of a Winston cone that directs the output energy toward the surface. The illumination source is a COTS IR-emitter consisting of a tungsten filament located 
inside an industry standard TO-8 package. The TO-8 package has an internal gold plated parabolic reflector and contains an inert gas sealed inside by a sapphire window.

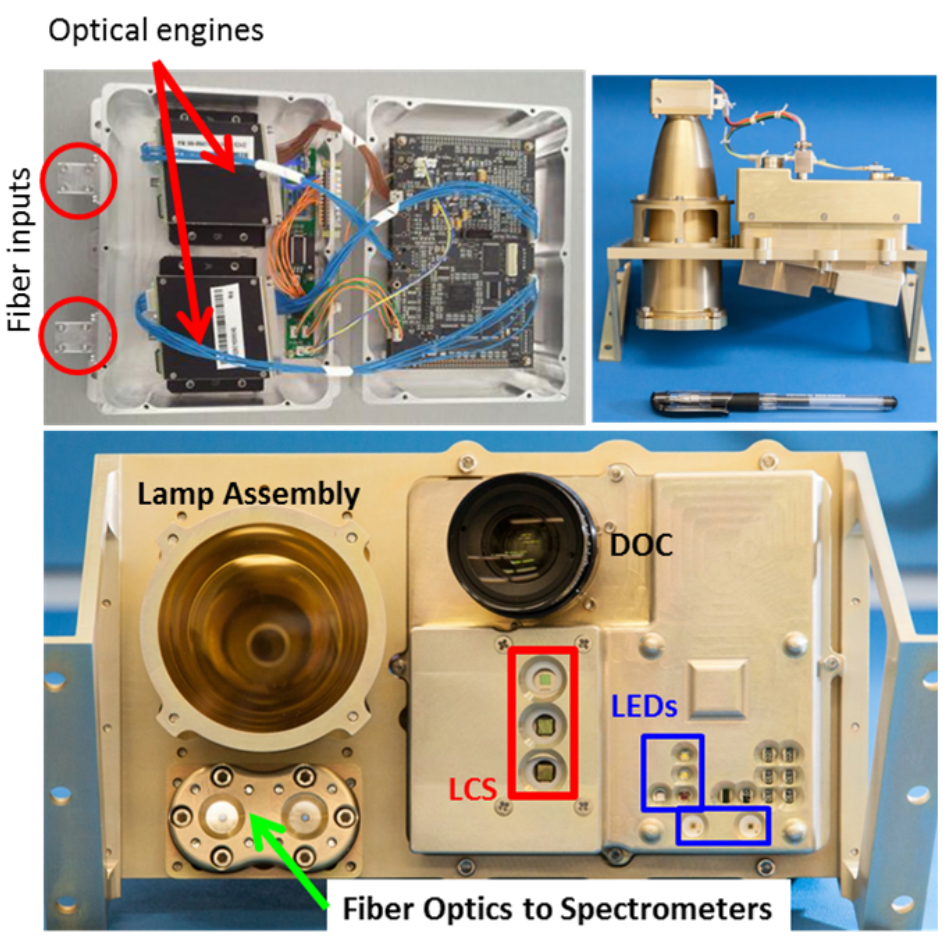

Figure 1 NIRVSS Spectrometer box. Top left shows fiber optic cable inputs to each optical engine. Top right is a side view of NIRVSS bracket assembly. Bottom is a view of NIRVSS bracket assembly with several components labeled.

Table 1. DOC Imaging scaling, volumes, and times

\begin{tabular}{|c|c|c|c|}
\hline Scale & Image, pixels & $\begin{array}{c}\text { Volume, } \\
\text { Mbytes }\end{array}$ & $\begin{array}{c}\text { Acquisition, read-out, } \\
\text { and transfer time }\end{array}$ \\
\hline 0 & $2048 \times 2048$ & 8.388 & 13 min. \\
\hline 1 & $1024 \times 1024$ & 2.097 & $3.25 \mathrm{~min}$. \\
\hline 2 & $512 \times 512$ & 0.524 & $43 \mathrm{sec}$. \\
\hline 3 & $256 \times 256$ & 0.129 & 9 sec. \\
\hline 4 & $128 \times 128$ & 0.033 & $\sim 4$ sec. \\
\hline 5 & $64 \times 64$ & 0.008 & $<1$ sec. \\
\hline
\end{tabular}

\section{GRC VF13 Chamber}

Only a brief summary of the GRC VF13 chamber is provided here and the interested reader is referred to Kleinhenz et al ${ }^{8,9}$ for more details. The VF13 is a cylindrical chamber able to accommodate aluminum tubes $(1.2 \mathrm{~m}$ tall and $25.4 \mathrm{~cm}$ internal diameter) containing regolith simulants. The chamber can reach pressures on the order of $10^{-6}$ Torr and active cooling of the simulants is achieved via liquid nitrogen. Three vertically spaced temperature probes are located within the simulant tube and each probe has five radially distributed thermocouples for characterizing the simulant temperature. A residual gas analyzer (RGA) operates at pressures $<10^{-4}$ Torr $^{9}$ and is used to monitor the gases evolved during drilling activities. The RGA provides pressure data for atomic masses of 1 to 50. Internally mounted video cameras are available to monitor the simulant during chamber activities.

3. Drill

A very terse summary of the RP drill is provided here. The RP drill is based upon the Icebreaker drill design, with some modifications, and the interested reader is referred to Zakny et al. ${ }^{4,10}$. The drill telemetry most relevant here is the date, time, depth of the drill, and drill bit temperature (measured by a thermocouple located $2.54 \mathrm{~cm}$ above the drill bit tip). Due to configurations necessary to accommodate both the drill and NIRVSS in the VF13 chamber, the maximum drill depth was constrained to about $40 \mathrm{~cm}$.

4

American Institute of Aeronautics and Astronautics 


\section{B. Experimental}

During March 2015 the NIRVSS ETU, Honeybee Robotics drill, and five sample capture mechanisms ${ }^{9}$ were placed in the GRC VF13 facility along with prepared lunar simulant soil tubes doped with varying amounts of water (Fig. 2). The objective was to simulate observations during drilling activities on the cold lunar surface and to assess

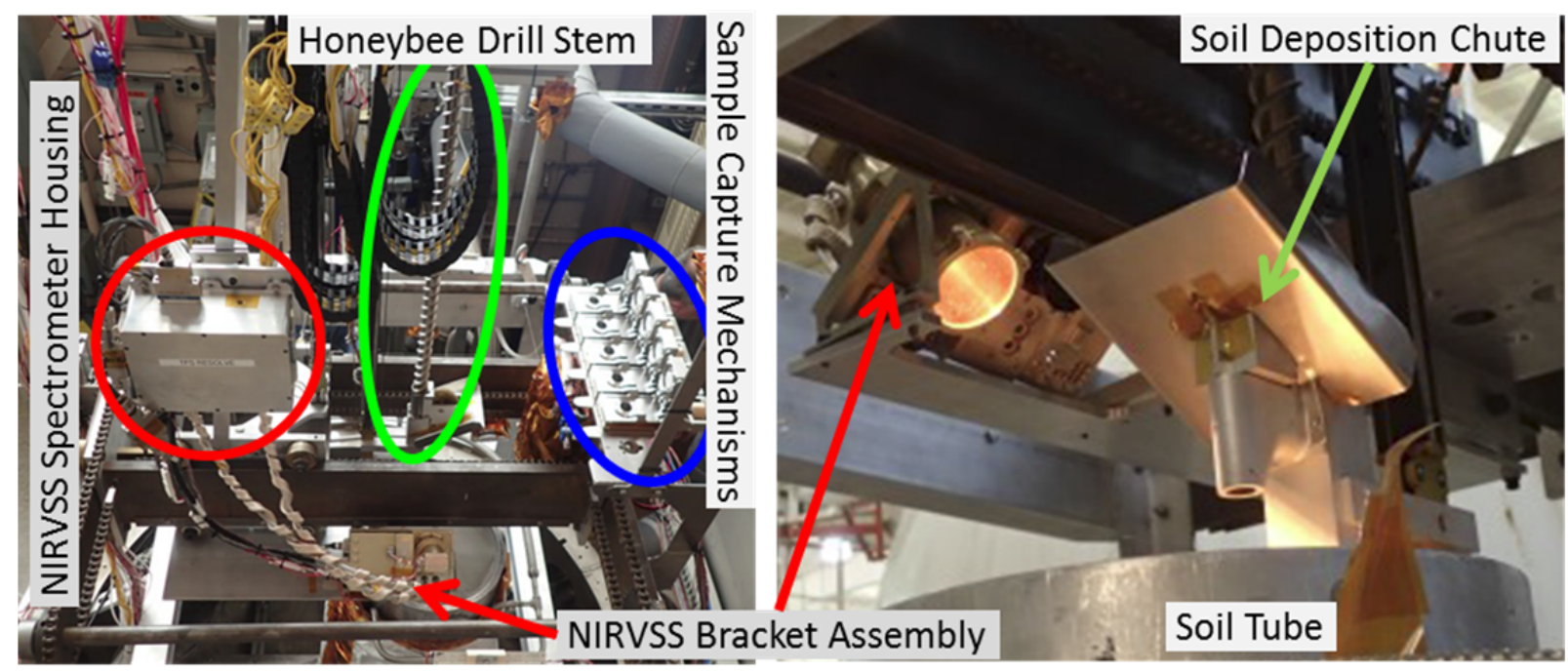

Figure 2 NIRVSS components mounted in GRC chamber. Left panel shows NIRVSS components (red arrows and circle) along with Honeybee Drill (green ellipse) and sample capture mechanisms (blue ellipse). Right panel shows NIRVSS bracket assembly looking downward toward soil tube where extracted soil will be deposited onto the surface by the drill.

effective delivery of soil samples for capture and sealing in the sample capture mechanisms. A brief summary of the experimental arrangements and associated instrumental measurements is provided here. Soil tube preparation is similar to that described by Kleinhenz et al. ${ }^{9}$. Two separate aluminum tubes were filled with lunar highlands simulant (NU-LHT-3M) having layers of differing water content. Both tubes had a dry-wet-dry stratigraphy, with layers separated by aluminum foil barriers. Due to vertical space constraints imposed by accommodating NIRVSS and the Honeybee drill, the drill only sampled the upper two layers. The boundary between the upper dryer layer and underlying wetter layer was approximately $16 \mathrm{~cm}$ from the top soil surface for both tubes. ST1 and ST2 were prepared with dry layers of $\sim 0.1$ and $\sim 0.5$ wt. $\%$ water, and underlain by a layer with $\sim 4.9$ wt. $\%$ water (Fig. 3 , top). The STs were placed in the chamber on different days, ST1 was observed on 9 March and ST2 on 16 March. After each tube was situated in the chamber, a thermal shroud was placed surrounding all components, the chamber was sealed and evacuation began and extended for a period of $\sim 48$ hours. After sealing the chamber, liquid nitrogen flowed through coils surrounding the STs providing cooling of the soils ${ }^{9}$. The shroud was maintained at $223^{\circ} \mathrm{K}$ and $143^{\circ} \mathrm{K}$ for ST1 and ST2, respectively, to simulate lunar conditions in sunlight and shadow. The thermocouples located in the soil tubes provided monitoring of the soil temperature, and average soil temperatures were $203^{\circ}$ to $213^{\circ} \mathrm{K}$ for ST1 and approximately $93^{\circ} \mathrm{K}$ for ST2.

Five holes were drilled in each soil tube in a sequence to keep the most recent cuttings pile within the NIRVSS instrumental FOVs (Fig. 3, bottom). In each hole, a sequence of increasing $10 \mathrm{~cm}$ drilling depths (pecks, or bites) occurred (Fig. 3, top). This sequence was 0-10 cm (peck 1, P1), 10-20 cm (peck 2, P2), 20-30 cm (peck 3, P3), and $30-40 \mathrm{~cm}$ (peck 4, P4) with the sample delivered to a SCN after P4. The process of drilling using pecks has many advantages that include preservation of stratigraphy, and lower drilling power and energy ${ }^{10}$. After P1, P2, and P3, the drill was extracted to $12 \mathrm{~cm}$ above the soil surface. During drilling and extraction drill cuttings were deposited onto the surface into a pile that typically grew with drilling depth. NIRVSS obtained images and spectra of this pile during the entire drilling sequence in each of the five holes. In order to provide an independent estimate of the soil surface temperature, a modification of the peck sequence was implemented for ST2. For P1 of each hole, drilling was halted at a depth of $2.5 \mathrm{~cm}$ for two minutes to permit the drill bit thermocouple to reach equilibrium with the soil surface. Then drilling activity proceeded as in ST1.

After activity with a soil tube was completed, the vacuum chamber was back-filled with nitrogen gas and returned to ambient pressure and temperature. After removal of the soil tube from the chamber, several samples 
were acquired and the soil moisture profile (Fig. 3, top) was determined using the approach described by Kleinhenz et al. ${ }^{9,11}$.

C. DOC image size impact on spectral data acquisition

During the GRC tests it was not possible to obtain simultaneous DOC images and spectra. As shown in Table 1 the higher resolution images, scales of 0 and 1, impose a significant delay in collecting spectra and impact the ability to monitor materials as they are deposited and exposed on the surface. Additionally, the imaging sequence automatically collected three images at different exposure times; imposing an even longer delay. As a result, these highest resolution images were only obtained prior and subsequent to drilling activities.

Images at scale $2(512 \times 512)$ were obtained at the beginning of ST1, Hole 1 (H1), P1. However, it was evident that spectral data collection still suffered. For the remainder of $\mathrm{H} 1$ a scale of 4 (128 $x$ 128) was used. For Hole 2 (H2) a scale of 3 (256 $x$ 256) was used, but again it became evident that spectral data collection suffered and for the remaining holes in ST1 an image scale of 4 was used. During active drilling of ST2, all images were obtained at an image scale of 4 . Figure 4 shows the instrumental FOVs of the unobscured soil tube relative to the DOC FOV with the IR emitter on (Fig. 4a) and with only the white LED (Fig. 4b). The location of the drill foot and soil chute, relative to these FOVs are shown in Fig. 4c.
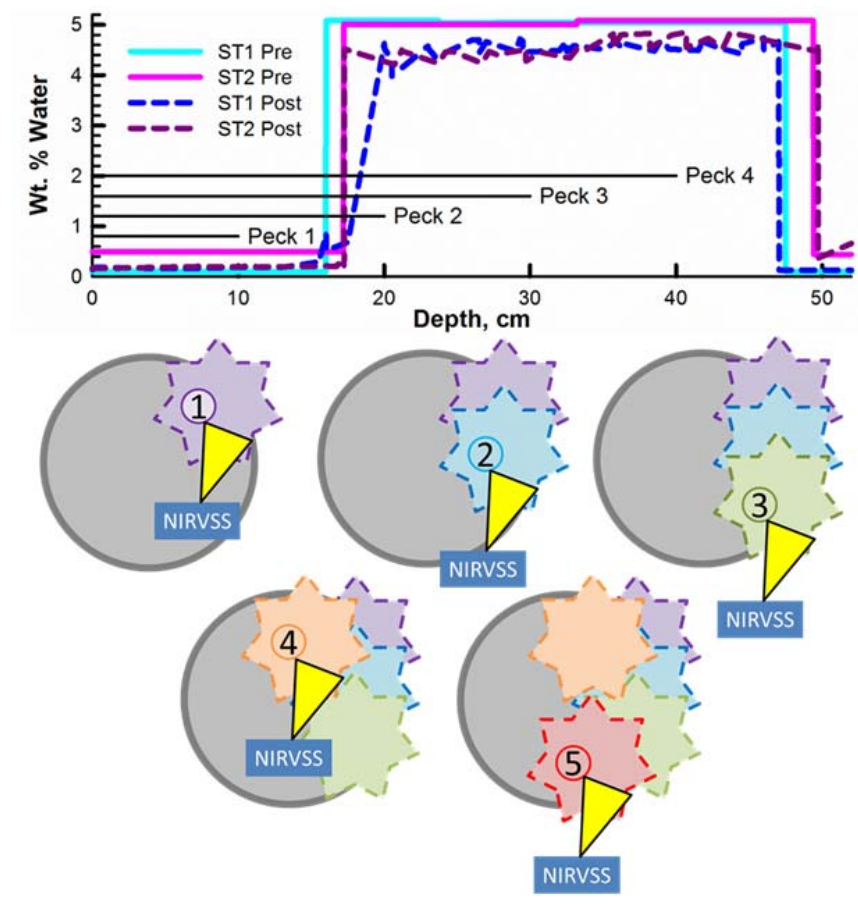

Figure 3 Water profiles and hole patterns. Top panel shows the soil water profiles for Soil Tubes 1 and 2 (ST1 and ST2 respectively) pre- (thick solid lines) and postemplacement (thick dashed lines) in the vacuum chamber. The thin black lines show the maximum penetration depth for each peck in each hole. Bottom panel is a cartoon illustrating the nominal position and sequence, e.g. 1 is the first hole, of the five holes drilled into the soil tubes. A notional cuttings piles and NIRVSS viewing geometry are illustrated by the star pattern for each hole and the triangle, respectively.
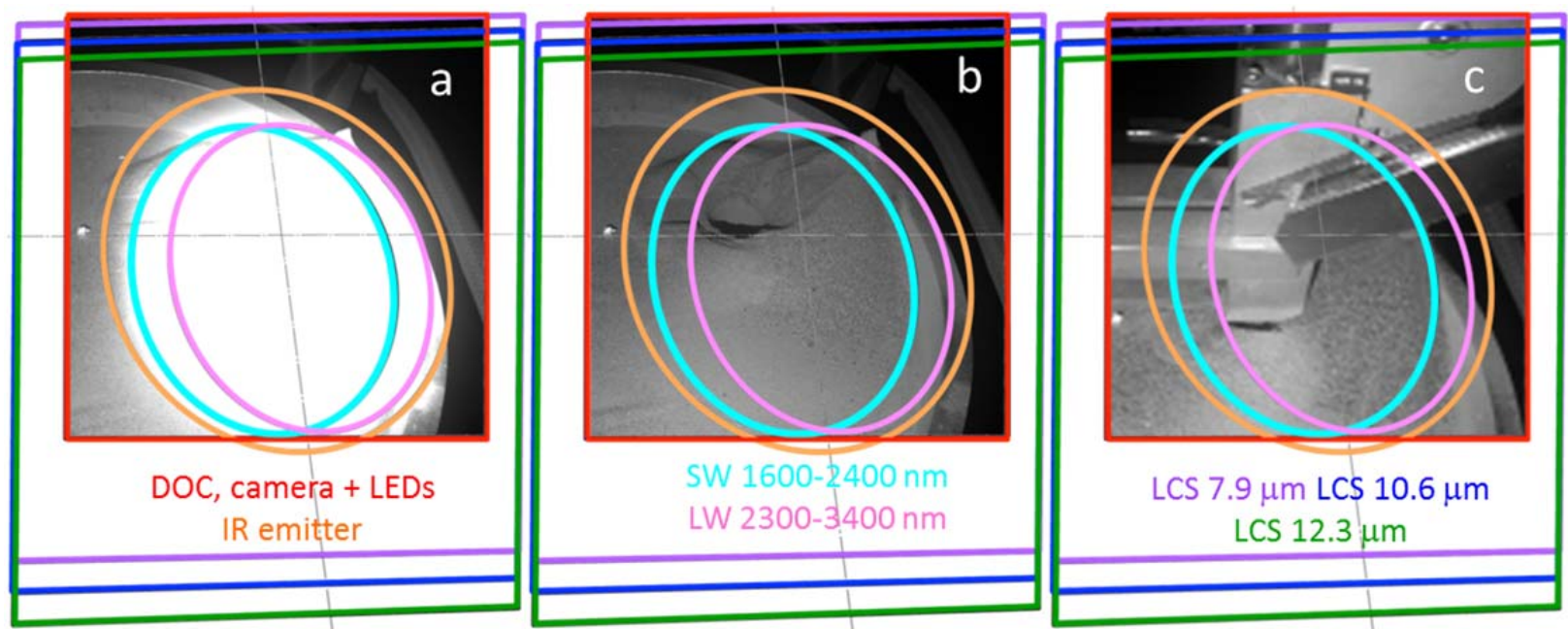

Figure 4 NIRVSS instrument FOV from the DOC perspective. (a) unobscured drill tube with the IR-emitter on; no LEDs, (b) only white LED, and (c) NIRVSS FOVs with the drill foot on the surface. 


\section{Data Analysis}

\section{A. Spectral Manipulation}

Spectrometer DNs are converted to radiances using laboratory reference measurements. However, this step is not particularly important for the interpretations presented here as spectra from different periods of activity are all divided by spectra obtained at the beginning of the data collection sequence for each hole in the tube. Thus, what is shown here is a measure of the spectral changes relative to pre-drilling activity at the beginning of each hole.

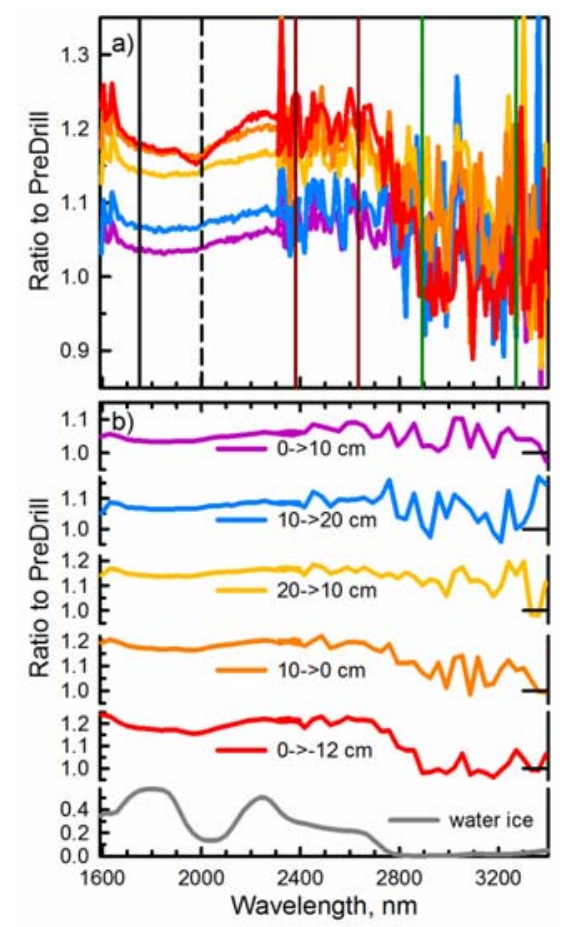

Figure 5 NIRVSS spectral ratios from DH1, P2. a) without smoothing, colored lines and wavelengths, or regions, used to characterize water ice spectral features (vertical lines). b) 5 point Lowess smoothing of panel a labeled with depth range, colors correspond to the curves in panel a. The right horizontal line in the top 5 panels are at a value of 1 . Bottom panel gray line is a spectrum of narticulate water ice.
Individual NIRVSS spectra, particularly the LW data, exhibit a peakto-peak variability that can represent a challenge for real-time identification of volatile spectral features. To improve the ability to identify such features, several spectra are averaged. For the data presented here the number of spectra in an average is typically about ten, but extremes range from three to over a hundred. At the beginning of each DH, for each ST, a reference spectrum is created by averaging all the spectra with the drill foot in the NIRVSS FOV, but prior to any drilling activity. During drilling activities averages were created for each $10 \mathrm{~cm}$ peck with some minor exceptions. Averages were also calculated while the drill was located at $12 \mathrm{~cm}$ above the surface, and also during the drill transition from $12 \mathrm{~cm}$ above the surface to $0 \mathrm{~cm}$ depth. For ST2, the $0-10 \mathrm{~cm}$ average was broken into two segments, $0-2.5 \mathrm{~cm}$ and $2.5-10 \mathrm{~cm}$, that reflect the pause in the drilling sequence at $2.5 \mathrm{~cm}$ depth.. For each $\mathrm{DH}$, these $\sim 10 \mathrm{~cm}$ spectral averages are divided by the average of the pre-drill spectra. This ratio highlights spectral differences occurring during the drill depth range from the initial placement of the drill foot. Figure 5a provides an example of these spectral ratios.

In order to more clearly document the behavior of the $3000 \mathrm{~nm}$ water ice band we investigated smoothing both the SW and LW data in wavelength. Using a commercial software program we performed 3,5 , and 7 point Lowess smoothing ${ }^{12,13}$ of each peck average and the reference spectra. Based upon visual analyses, and trading off peakto-peak variability with retention of spectral features, we selected a 5 point Lowess smoothing in wavelength for all spectral ratios shown in the multiple panels of Figure 5b. The laboratory spectrum of water ice is provided as a reference in the bottom panel of Fig. $5 \mathrm{~b}$.

\section{B. Band parameters}

NASA's RP mission is intended to operate with decisions regarding sample acquisition and evolved gas analyses made in realtime. While the spectral manipulation discussed above is appropriate for more detailed analyses, it is time-consuming and likely would not be amenable for providing input into real-time decision-making.

To overcome potential time delays we use spectral band ratios to characterize the diagnostic spectral features of volatile materials. The NIRVSS SW spectrometer samples the 2000 $\mathrm{nm}$ spectral feature of water ice. To characterize this feature we select two radiance values at a wavelength centered at $2001 \mathrm{~nm}$ (Fig. 5a dashed black line, $\mathrm{r}_{2001}$ ) and another wavelength outside the spectral feature at $1751 \mathrm{~nm}$ (Fig. 5a solid black line, $\left.\mathrm{r}_{1751}\right)$. Using these values for individual spectra, not averages, we calculate the band depth $\left(\mathrm{BD}_{2000}\right)$ from the expression of Clark and Roush ${ }^{14} ; \mathrm{BD}_{2000}=1-\left(\mathrm{r}_{2001} / \mathrm{r}_{1751}\right)$. The NIRVSS LW spectrometer samples the much stronger fundamental of water ice near $3000 \mathrm{~nm}$ and hence is potentially more sensitive to lower abundances. Because of the higher variability of the individual spectra in the LW spectrometer, we use average of radiances over wavelengths rather than a single wavelength in the band depth $\left(\mathrm{BD}_{3000}\right)$ calculation. The band depth expression becomes $\mathrm{BD}_{3000}=1-\left[\operatorname{avg}\left(\mathrm{r}_{(2793: 3271)}\right) / \operatorname{avg}\left(\mathrm{r}_{(2381: 2635}\right)\right]$ where the subscript refers to the beginning and ending wavelength used in the average. The ranges in the numerator and denominator lie between the vertical dark green and red lines in Fig. 5a, respectively. We also calculate the mean and standard deviation of these two band depths prior to the beginning of drilling in DH1.

American Institute of Aeronautics and Astronautics 


\section{Results}

NIRVSS spectra clearly show the appearance of water ice as the drilling proceeds for both soil tubes. The features near $3000 \mathrm{~nm}$ are most sensitive to water, but weaker spectral features near $2000 \mathrm{~nm}$ were readily observed during portions of the drilling sequence. As shown in Figures 5 and 6, the strengths of these features vary as a function of drill depth, both increasing and decreasing as the drill excavates and extracts differing materials. The strengths of these features also appear to temporally correlate with mass 18 fluctuations $\left(\right.$ i.e. $\left.\mathrm{H}_{2} \mathrm{O}\right)$ monitored independently via the RGA (Fig. 6). Differences seen for the same peck, but in a different hole are also observed. For example in the bottom panel of Fig. 6 both calculated band depths are much weaker for peck 3 of hole 5 .

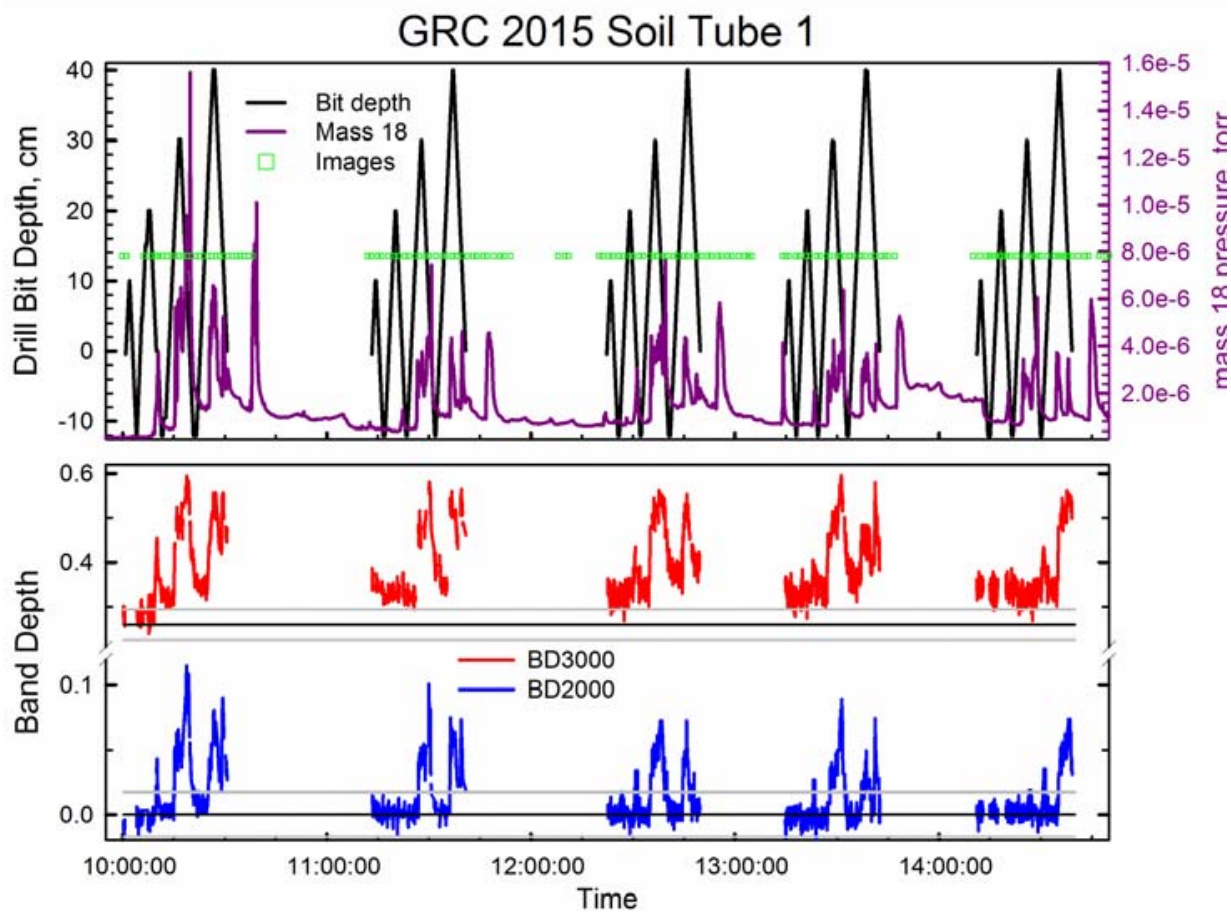

Figure 6 Measurements as a function of time. Top panel: drill bit depth (black), mass 18 pressure $\left(\mathrm{H}_{2} \mathrm{O}\right.$, purple), and DOC images (green squares). Each group of four bit depth peaks corresponds to the 4 pecks within a single hole. Bottom panel: calculated $2000 \mathrm{~nm}$ (blue) and $3000 \mathrm{~nm}$ (red) band depths. Average band depths, prior to drilling (black lines), with their \pm 2 standard deviations are shown (gray lines). Gaps represent soil delivery to the sample capture mechanisms.

NIRVSS DOC images show that the relatively homogeneous simulant exhibits albedo variations that may be associated with varying water content and pieces of the aluminum foil barriers are readily observed (Fig. 7 arrow top left panel). The DOC images document cuttings pile growth and occasional collapse. Within the confined space of the experimental configuration, Figure 7 illustrates the DOC also documented filling of an existing hole which results in inconsistent spectral behavior for sampling the same depth in different holes (Fig. 7 right column). This infilling explains the apparent anomalous behavior of the band depths for DH5, P2-P3 seen in Fig. 6.

\section{Conclusions}

Monitoring drilling activities into a water-doped lunar simulant in a cryogenic, vacumn environment with NIRVSS documents compositional and morphological changes in real-time. The NIRVSS spectra exhibit water ice spectral features that vary as differing concentrations of the simulant are exposed and collect on the surface. Spectral parameters associated with the water ice features exhibit temporal trends consistent with the increased pressure of mass 18, e.g., water, measured by the RGA. Additionally, DOC images provide valuable information documenting the physical nature of the drilling activity and thus the physical properties of the materials encountered. A few examples of such physical properties include the angle of repose, granular and cohesive nature of surface and sub-surface materials, and surface compaction due to the drill foot. This information could be cross-correlated with 

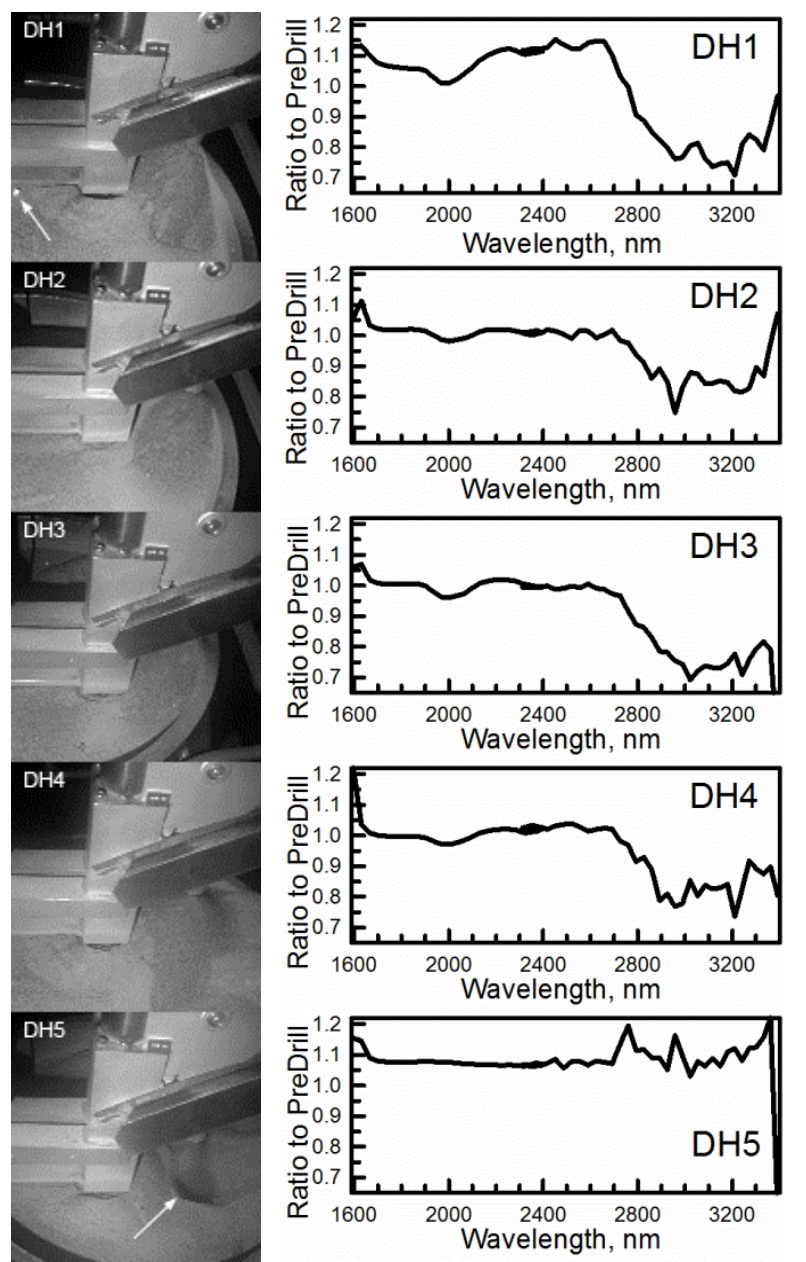

the drilling telemetry for the same depth interval. Some anomalous spectral behavior was explained by temporally correlating NIRVSS spectra with DOC images.

NIRVSS is able provide insight into the surface and sub-surface volatile content during drilling activities. This insight can be used as part of a decision process to determine whether or not to deliver a sample for more detailed analyses by other rover instruments.

\section{Acknowledgments}

TLR and AMC thank NASA's Human Exploration and Operations Mission Directorate for their continued support of NIRVSS development.

\section{References}

${ }^{1}$ Andrews, D., A. Colaprete, J. Quinn, D. Chavers, and M. Picard (2014) Introducing the Resource Prospector (RP) Mission, 52nd AIAA 2014 Space Conference and Exposition, 47 August 2014, San Diego, CA, doi: 10.2514/6.2014-4378.

${ }^{2}$ Elphic, R. and 10 others (2015) Simulated real-time lunar volatiles prospecting with a rover-borne neutron spectrometer, Adv. Sp. Res., 55, 2438-2450, doi:10.1016/j.asr.2015.01.035.

${ }^{3}$ Roush, T.L., and 9 others (2015) Attempt of serendipitous science during the Mojave Volatile Prospector field expedition, 46th Lunar Plant. Sci. Conf., 16-20 March 2015, The Woodlands, TX abstract 1956.

${ }^{4}$ Zacny K., G. Paulsen, P. Chu, B. Mellerowicz, B. Yaggi, J. Kleinhenz, and J. Smith (2015). The Icebreaker Drill System: Sample acquisition and delivery for the lunar Resource Prospector Mission, 46th Lunar Planet. Sci. Conf., 16-20 March 2015, The Woodlands, TX, abstract 1614.

${ }^{5}$ Paz, A., L. Oryshchyn, S. Jensen, G.B. Sanders, K. Lee, and M. Reddington (2013) RESOLVE OVEN field demonstration unit for lunar resource extraction, 51st Aerospaces Science Meeting and Exposition, 7-10 January 2013, Grapevine, TX, doi: 10.2514/6.2013-734.

${ }^{6}$ Captain, J. and 10 others (2015) Design and development of volatile analysis system for analog field test of lunar exploration mission, Adv. Sp. Res., 55, 2457-2471, doi:10.1016/j.asr.2014.11.006.

${ }^{7}$ Ennico, K., M. Shirley, A. Colaprete, and L. Osetinsky a previous DH (left column, bottom panel arrow).

(2012) The Lunar Crater Observation and Sensing Satellite (LCROSS) payload development and performance in flight, Sp. Sci. Rev., 187, 23-69, doi: 10.1007/s11214-011-9753-4

${ }^{8}$ Kleinhenz, J. (2014) Lunar polar environmental eesting: regolith simulant conditioning, AIAA SciTech, 7th Symposium on Space Resource Utilization, 13-17 January 2014, National Harbor, MD.

${ }^{9}$ Kleinhenz, J., G. Paulsen, K. Zacny, and J. Smith (2015) Impact of drilling operations on lunar volatiles capture: thermal vacuum tests. AIAA SciTech 2015: 53rd Aerospace Sciences Meeting and Exhibit, 5-9 January 2015, Kissimmee, FL, AIAA, doi: 10.2514/6/2.2015-1177.

${ }^{10}$ Zacny K., G. Paulsen, C.P. McKay, B. Glass, A. Davé, A.F. Davila, M. Marinova, B. Mellerowicz, J. Heldmann, C. Stoker, N. Cabrol, M. Hedlund, and J. Craft (2013). Reaching $1 \mathrm{~m}$ Deep on Mars: The Icebreaker Drill, Astrobiology, 13, $1166-1198$. doi:10.1089/ast.2013.1038.

${ }^{11}$ Kleinhenz, J., G.Paulsen, K. Zacny, S,K, Schmidt, and D.S. Boucher (2016) Regolith volatile recovery at simulated lunar environment, AIAA SciTech 2016, 4-8 January 2016, San Diego, CA.

${ }^{12}$ Cleveland, W. S. (1979). Robust locally weighted regression and smoothing scatterplots, J. Am. Statistical Assoc., 74, 829836, doi: $10.2307 / 2286407$.

${ }^{13}$ Cleveland, W.S., and S.J. Devlin (1988). Locally-weighted Regression: an approach to regression analysis by local fitting, J. Am. Statistical Assoc., 83 (403): 596-610, doi:10.2307/2289282.

14 Clark, R.N. and T.L. Roush (1984) Reflectance spectroscopy: Quantitative analysis techniques for remote sensing applications, J. Geophys. Res. 89, 6329-6340. 